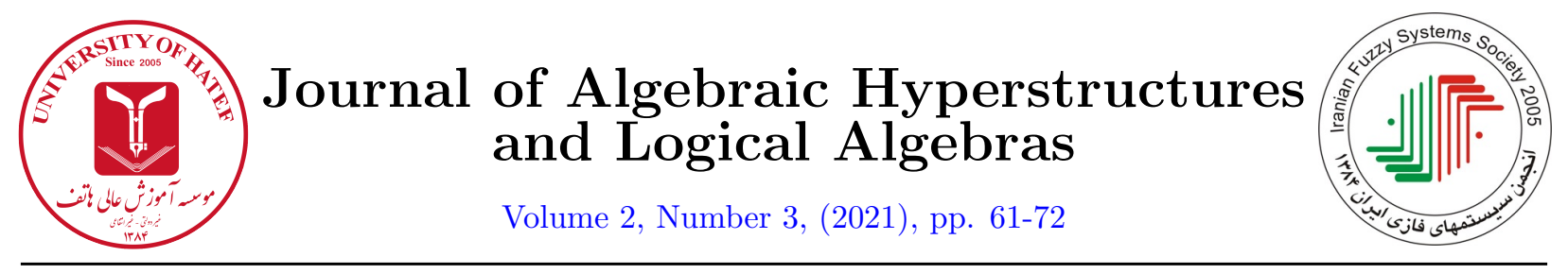

\title{
Cubic soft matrices with applications in medical diagnosis
}

\author{
M. Khan ${ }^{1}$, S. Anis ${ }^{2}$ and S. Iqbal ${ }^{3}$ \\ ${ }^{1,2}$ Department of Mathematics, COMSATS University Islamabad, Abbottabad Campus, Pakistan \\ ${ }^{3}$ Department of Mathematics, COMSATS University Islamabad, Islamabad Campus, Pakistan
}

madadmath@yahoo.com, saimaanispk@gmail.com, soh.iqbal@gmail.com

"This paper is dedicated to Professor Young Bae Jun on the occasion of his 70th birthday."

\begin{abstract}
The purpose of this paper is to introduce and explore cubic soft matrix theory. Moreover we apply the notion of cubic soft matrices using the weighted arithmetic means for applications in medical diagnosis by introducing two algorithms.
\end{abstract}

\section{Article Information}

Corresponding Author:

M. Khan;

Received: March 2021;

Accepted: Invited paper;

Paper type: Original.

\section{Keywords:}

Cubic set, cubic soft set, cubic soft matrix.

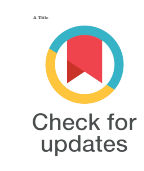

\section{Introduction}

There are a lots of complications, uncertainties and inexactness in models of all branches of science. Most of these models are related to human life. Several methods and techniques are introduced for handling such ambiguities. One of the fundamental concept among these techniques, was introduced by L. A. Zadeh [24] in 1965. He established the idea of a fuzzy set to handle these complications, which is the extension of the classical notion of a crisp set. A fuzzy set is characterized by a membership function $\mu$ within the range $[0,1]$. In a fuzzy set, the truth membership value of an element is a single value between $[0,1]$. However in reality, it may not always be true that the degree of non-membership value of an element in a fuzzy set is equal to 1 minus the membership value because there may be some hesitation degree. Fuzzy set theory has many applications in major fields such as engineering, control engineering, operation research, management science, robotics and much more. There are many published papers on a fuzzy set which shows its importance and its applications to the set theory, algebra, real analysis, measure theory and topology.

An intuitionistic fuzzy set was introduced by K. Atanassov [I] (1983) which is the extension of Lotfi Zadeh's notion of a fuzzy set. An intuitionistic fuzzy set is the generalization of fuzzy set. The elements of an intuitionistic fuzzy set have degree of memberships (as in fuzzy set) range in $[0,1]$ as well as the degree of non-membership range in $[0,1]$. Thus the intuitionistic fuzzy set is characterized by a membership function $\mu$ and non-membership function by $\nu$ with range $[0,1]$ such as yes or no (in voting system), winning or

https://doi.org/10.52547/HATEF.JAHLA.2.3.5 
loosing (in a game), right or wrong (in making a decision) etc. De et, al., (2001) [8] gave an intuitionistic fuzzy set approach in medical diagnosis using three steps such as; determination of symptoms, formulation of medical knowledge based on intuitionistic fuzzy relations, and determination of diagnosis on the basis of composition of intuitionistic fuzzy relations. An intuitionistic fuzzy set is a tool which is used for modelling real life problems like sale analysis, new product marketing, financial services and negotiation process.

The data is not always crisp in many complicated problems of many fields as: Economics, engineering, environment, social science, medical science, etc. Since uncertainties are always possesses in these problems, therefore we cannot always use the classical methods. There are many theories, viz., theory of probability,

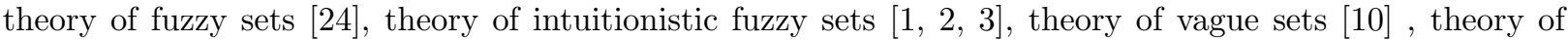
interval mathematics [3, 5], and theory of rough sets [18] which can be used as mathematical tools to handle the uncertainties. But since there are many inherent difficulties in these theories, there was a need of a new concept for handling such difficulties. One of a new concept called soft set was introduced by Molodtsov in 1999 [16] as a general mathematical tool to overcome the problems related to uncertainties. The soft set theory was applied by Molodtsov successfully in several directions, such as smoothness of functions, operations research, probability, game theory, Riemann integration, Perron integration, theory of measurement and so on. Chen et al., [7] and Xiao et al. [2I] presented research on synthetically evaluating a method for business competitive capacity based on soft sets. Maji et. al., [15] gave a practical application of soft sets in decision making problems.

Soft matrix was introduced by Cagman et. al., [5] in 2010 which is the representation of a soft set, to define more operations in theoretical studies in soft sets. This representation has many advantages and applications in decision sciences.

The concept of fuzzy soft sets was introduced by Maji at. al., [14] in 2001 by combining soft sets and fuzzy sets with some properties viz fuzzy soft union, intersection, complement of a fuzzy soft set, De Morgan's Law etc. Yang et. al., [2:3] defined the generalization of fuzzy soft sets and evaluated a decision making problem by using fuzzy soft sets. Mostly researchers have been studied the applications of fuzzy soft set theory in many real life situations (circumstances).

Yang [22] and C. Ji [22], defined fuzzy soft matrix (FSM) in 2011, which represents and calculate the data involving fuzzy soft sets. Cagman $[\overline{6}]$ and Enginoglu defined fuzzy soft matrices and constructed a decision making problem. Fuzzy soft matrix theory and its application extended by Borah et. al., [4].

Intuitionistic fuzzy parameterized soft sets were introduced by Deli [9] and Cagman. They used intuitionistic fuzzy parameterized soft sets to solve uncertainties based problems. Babitha [3] and John [3] introduced the concept of generalized intuitionistic fuzzy soft sets which are applied in multi criteria decision making problems. Rajarajeswari [[.9] and Dhanalakshmi [[.9] worked on intuitionistic fuzzy soft matrix and their operations.

Cubic soft sets were introduced by Muhiuddin and Al-roqi [17]. They introduced the concept of (external, internal) cubic soft sets, P-cubic (resp., R-cubic) soft subsets, R-union (resp., R-intersection, P-union and P-intersection) of cubic soft sets and the complement of a cubic soft set. They also described some properties which are applied on cubic soft sets to BCK/BCI-algebras.

\section{Preliminaries}

Definition 2.1. Let $U$ be an initial universe, $P(U)$ be the power set of $U, E$ be the set of all parameters and $A \subseteq E$. A soft set $\left(f_{A}, E\right)$ on the universe $U$ is defined by the set of order pair

$$
\left(f_{A}, E\right)=\left\{\left(e, f_{A}(e)\right): e \in E, f_{A}(e) \in P(U)\right\},
$$

where $f_{A}: E \rightarrow P(U)$ such that $f_{A}(e)=\phi$ if $e \notin A$.

Here $f_{A}$ is called an approximate function of the soft set $\left(f_{A}, E\right)$. The set $f_{A}(e)$ is called an e-approximate value set or an e-approximate set which consists of related objects of the parameter $e \in E$.

Example 2.2. Let we have a set of five shirts $W=\left\{w_{1}, w_{2}, w_{3}, w_{4}, w_{5}\right\}$ and a set of parameters such as $V=\left\{\operatorname{red}\left(v_{1}\right), \operatorname{pink}\left(v_{2}\right)\right.$, purple $\left.\left(v_{3}\right)\right\}$. Let $A=\left\{v_{1}, v_{2}\right\}, A \subseteq V, f_{A}\left(v_{1}\right)=\left\{w_{1}, w_{2}, w_{3}, w_{4}\right\}$ and $f_{A}\left(v_{2}\right)=\left\{w_{1}, w_{2}, w_{3}\right\}$. Then $\left(f_{A}, V\right)=\left\{\left(v_{1},\left\{w_{1}, w_{2}, w_{3}, w_{4}\right\}\right),\left(v_{2},\left\{w_{1}, w_{2}, w_{3}\right\}\right)\right\}$ is the soft set over $W$ which represents the "colour of the shirts" which Mr. X is going to buy. 
Definition 2.3. Let $U$ be an initial universe, $E$ be the set of all parameters and $A \subseteq E$. A pair $(F, A)$ is called a fuzzy set over $U$ where $F: A \rightarrow P(U)$ is a mapping from $A$ into $P(U)$, where $P(U)$ denotes the collection of all subsets of $U$.

Example 2.4. Consider the Example [2.9, here we can not express with only two real numbers 0 and 1, we can characterized it by a membership function instead of crisp number 0 and 1, which associate with each element a real number in the interval $[0,1]$. Then

$$
\left(f_{A}, V\right)=\left\{f_{A}\left(v_{1}\right)=\left\{\left(w_{1}, 0.3\right),\left(w_{2}, 0.6\right),\left(w_{3}, 0.5\right),\left(w_{4}, 0.2\right)\right\}, f_{A}\left(v_{2}\right)=\left\{\left(w_{1}, 0.4\right),\left(w_{2}, 0.7\right),\left(w_{3}, 0.5\right)\right\}\right\} .
$$

Definition 2.5. Let $\left(f_{A}, E\right)$ be a fuzzy soft set over $U$. Then a subset of $U \times E$ is uniquely defined by $R_{A}=\left\{(u, e): e \in A, u \in f_{A}(e)\right\}$, which is called relation form of $\left(f_{A}, E\right)$. The characteristic function of $R_{A}$ is written by $\nu_{R_{A}}: U \times E \rightarrow[0,1]$, where $\nu_{R_{A}}(u, e) \in[0,1]$ is the membership value of $u \in U$ for each $e \in E$. If $\nu_{i j}=\nu_{R_{A}}\left(u_{i}, e_{j}\right)$, then we can define a matrix

$$
\left[\nu_{i j}\right]_{m \times n}=\left[\begin{array}{llll}
\nu_{11} & \nu_{12} & \cdots & \nu_{1 n} \\
\nu_{21} & \nu_{22} & \cdots & \nu_{2 n} \\
\vdots & \vdots & \vdots & \vdots \\
\nu_{m 1} & \nu_{m 2} & \cdots & \nu_{m n}
\end{array}\right]
$$

which is called an $m \times n$ soft matrix of the soft set $\left(f_{A}, E\right)$ over $U$. Therefore we can say that a fuzzy soft set $\left(f_{A}, E\right)$ is uniquely characterized by the matrix $\left[\nu_{i j}\right]_{m \times n}$ and both concepts are interchangeable.

Example 2.6. Assume that $U=\left\{u_{1}, u_{2}, u_{3}, u_{4}, u_{5}, u_{6}\right\}$ is a universal set and $E=\left\{e_{1}, e_{2}, e_{3}, e_{4}\right\}$ is a set of all parameters. If $A=\left\{e_{1}, e_{2}, e_{3}\right\} \subseteq E$ and

$$
\begin{aligned}
& f\left(e_{1}\right)=\left\{\left(u_{1}, 0.2\right),\left(u_{2}, 0.1\right),\left(u_{3}, 0.5\right),\left(u_{4}, 0.3\right),\left(u_{5}, 0.7\right),\left(u_{6}, 0.9\right)\right\}, \\
& f\left(e_{2}\right)=\left\{\left(u_{1}, 0.9\right),\left(u_{2}, 0.3\right),\left(u_{3}, 0.8\right),\left(u_{4}, 0.4\right),\left(u_{5}, 0.6\right),\left(u_{6}, 0.2\right)\right\}, \\
& f\left(e_{3}\right)=\left\{\left(u_{1}, 0.6\right),\left(u_{2}, 0.4\right),\left(u_{3}, 0.2\right),\left(u_{4}, 0.5\right),\left(u_{5}, 0.3\right),\left(u_{6}, 0.8\right)\right\},
\end{aligned}
$$

then the fuzzy soft set $\left(f_{A}, E\right)$ is a parameterized family $\left\{f_{A}\left(e_{1}\right), f_{A}\left(e_{2}\right), f_{A}\left(e_{3}\right)\right\}$ of all fuzzy sets over $U$. Hence the fuzzy soft matrix $\left[\nu_{i j}\right]$ can be written as

$$
\left[\nu_{i j}\right]_{6 \times 4}=\left[\begin{array}{cccc}
0.2 & 0.9 & 0.6 & 0.0 \\
0.1 & 0.3 & 0.4 & 0.0 \\
0.5 & 0.8 & 0.2 & 0.0 \\
0.3 & 0.4 & 0.5 & 0.0 \\
0.7 & 0.6 & 0.3 & 0.0 \\
0.9 & 0.2 & 0.8 & 0.0
\end{array}\right] .
$$

Definition 2.7. Let $U$ be an initial universe, $E$ be the set of parameters and $A \subseteq E$. Suppose that $\left(f_{A}, E\right)$ is an intuitionistic fuzzy soft set (IFSS) over $U$. Then a subset of $U \times E$ is uniquely defined by $R_{A}=$ $\left\{(u, e): e \in A, u \in f_{A}(e)\right\}$, which is called relation form of $\left(f_{A}, E\right)$. The membership and non-membership functions of $R_{A}$ are given by $v_{R_{A}}: U \times E \rightarrow[0,1]$ and $\omega_{R_{A}}: U \times E \rightarrow[0,1]$ where $v_{R_{A}}(u, e) \in[0,1]$ and $\omega_{R_{A}}(u, e) \in[0,1]$ are the membership value and non-membership value of $u \in U$ for each $e \in E$, respectively. If $\left(v_{i j}, \omega_{i j}\right)=\left(v_{i j}\left(u_{i}, e_{j}\right), \omega_{i j}\left(u_{i}, e_{j}\right)\right)$, then we can define a matrix as

$$
\left[v_{i j}, \omega_{i j}\right]_{m \times n}=\left[\begin{array}{llll}
\left(v_{11}, \omega_{11}\right) & \left(v_{12}, \omega_{12}\right) & \cdots & \left(v_{1 n}, \omega_{1 n}\right) \\
\left(v_{21}, \omega_{21}\right) & \left(v_{22}, \omega_{22}\right) & \cdots & \left(v_{2 n}, \omega_{2 n}\right) \\
\vdots & \vdots & \vdots & \vdots \\
\left(v_{m 1}, \omega_{m 1}\right) & \left(v_{m 2}, \omega_{m 2}\right) & \cdots & \left(v_{m n}, \omega_{m n}\right)
\end{array}\right],
$$

which is called an $m \times n$ IFSM of IFSS $\left(f_{A}, E\right)$ over $U$. Therefore we can say that IFSS $\left(f_{A}, E\right)$ is uniquely characterized by the matrix $\left[v_{i j}, \omega_{i j}\right]_{m \times n}$ and both concepts are interchangeable. The set of all $m \times n$ IFS matrices are denoted by $\operatorname{IFSM}_{m \times n}$. 
Example 2.8. Let $U=\left\{u_{1}, u_{2}, u_{3}, u_{4}, u_{5}\right\}$ be a universal set and $E=\left\{e_{1}, e_{2}, e_{3}, e_{4}\right\}$ be a set of parameters. If $A=\left\{e_{1}, e_{2}, e_{3}\right\} \subseteq E$ and

$$
\begin{aligned}
& f_{A}\left(e_{1}\right)=\left\{\left(u_{1}, 0.2,0.3\right),\left(u_{2}, 0.1,0.4\right),\left(u_{3}, 0.3,0.6\right),\left(u_{4}, 0.7,0.5\right),\left(u_{5}, 0.8,0.9\right)\right\}, \\
& f_{A}\left(e_{2}\right)=\left\{\left(u_{1}, 0.1,0.5\right),\left(u_{2}, 0.4,0.2\right),\left(u_{3}, 0.7,0.3\right),\left(u_{4}, 0.4,0.8\right),\left(u_{5}, 0.9,0.1\right)\right\}, \\
& f_{A}\left(e_{3}\right)=\left\{\left(u_{1}, 0.5,0.4\right),\left(u_{2}, 0.7,0.6\right),\left(u_{3}, 0.3,0.8\right),\left(u_{4}, 0.2,0.7\right),\left(u_{5}, 0.1,0.4\right)\right\},
\end{aligned}
$$

then IFSS $\left(f_{A}, E\right)$ is a parametrized family $\left\{f_{A}\left(e_{1}\right), f_{A}\left(e_{2}\right), f_{A}\left(e_{3}\right)\right\}$ of all IFSS over U. Hence IFSM $\left[\left(v_{i j}, \omega_{i j}\right)\right]_{m \times n}$ can be written as

$$
\left[\left(v_{i j}, \omega_{i j}\right)\right]=\left[\begin{array}{rrrr}
(0.2,0.3) & (0.1,0.5) & (0.5,0.4) & (0,0) \\
(0.1,0.4) & (0.4,0.2) & (0.7,0.6) & (0,0) \\
(0.3,0.6) & (0.7,0.3) & (0.3,0.8) & (0,0) \\
(0.7,0.5) & (0.4,0.8) & (0.2,0.7) & (0,0) \\
(0.8,0.9) & (0.9,0.1) & (0.1,0.4) & (0,0)
\end{array}\right]
$$

Definition 2.9. Let $X$ be a nonempty set. By a cubic set in $X$ we mean a structure

$$
\mathfrak{A}=\{\langle x, A(x), \lambda(x)\rangle \mid x \in X\},
$$

in which $A$ is an interval valued fuzzy set (IVF set) in $X$ and $\lambda$ is a fuzzy set in $X$ which is denoted by $\mathfrak{A}=\langle A, \lambda\rangle$. Let us denote the collection of all cubic sets by $C^{U}$.

$A$ cubic set $\mathfrak{A}=\langle A, \lambda\rangle$, in which $A(x)=0$ and $\lambda(x)=1$ (resp. $A(x)=1$ and $\lambda(x)=0$ ) for all $x \in X$ is denoted by $\ddot{0}$ (resp. $\ddot{1})$.

$A$ cubic set $\mathfrak{B}=\langle B, \mu\rangle$, in which $B(x)=0$ and $\mu(x)=0$ (resp. $B(x)=1$ and $\mu(x)=1)$ for all $x \in X$ is denoted by $\hat{0}$ (resp. $\hat{1})$.

Cubic Soft Set: Let we have a universal set $U$ and the set of parameters $V$. If $W \subset V$, then the cubic soft set over $U$ is represented by a pair $(\delta, W)$, where $\delta=\left\langle\widetilde{\rho}_{\delta}, \sigma_{\delta}\right\rangle$ is a mapping given by $\widetilde{\rho}_{\delta}: W \rightarrow P\left(C^{U}\right)$ and $\sigma_{\delta}: W \rightarrow C^{U}$ where $C^{U}$ represents the set of all cubic subsets and $P\left(C^{U}\right)$ represents the power set of $C^{U}$.

Cube Soft Matrix: Let the set of universe be $U=\left\{p_{1}, p_{2}, p_{3}, \ldots, p_{n}\right\}$ and the set of parameters be $V=\left\{q_{1}, q_{2}, q_{3}, \ldots, q_{m}\right\}$. If $W \subset V$, then $(\delta, W)$ is a cubic soft set over $U$, where $\delta=\left\langle\widetilde{\rho}_{\delta}, \sigma_{\delta}\right\rangle$ is a mapping given by $\widetilde{\rho}_{\delta}: W \rightarrow P\left(C^{U}\right)$ and $\sigma_{\delta}: W \rightarrow C^{U}$ where $P\left(C^{U}\right)$ is the power set of $C^{U}$ and $C^{U}$ is the set of all cubic subsets. The matrix representation of cubic soft set $(\delta, W)$ is $\widetilde{M}_{n \times m}=\left[\left(\widetilde{a}_{i j}, a_{i j}\right)\right]_{n \times m}$ or simply $\widetilde{M}=\left[\left(\widetilde{a}_{i j}, a_{i j}\right)\right]$, for $i=1,2, \ldots, n$ and $j=1,2,3, \ldots, m$, which is called the cubic soft matrix, where

$$
\widetilde{a}_{i j}=\left\{\begin{array}{ll}
\widetilde{\rho}_{\delta}\left(q_{i}\right)_{j} & \text { if } q_{i} \in W \\
{[0,0]} & \text { otherwise }
\end{array},\right.
$$

and

$$
a_{i j}=\left\{\begin{array}{ll}
\sigma_{\delta}\left(q_{i}\right)_{j} & \text { if } q_{i} \in W \\
1 & \text { otherwise }
\end{array},\right.
$$

$\left(\widetilde{\rho}_{\delta}\left(q_{i}\right)_{j}, \sigma_{\delta}\left(q_{i}\right)_{j}\right)$ represents the element of $\widetilde{M}$ corresponds to element $p_{i}$ of $U$, for $i=1,2,3, \ldots, n$, where $\widetilde{\rho}_{\delta}\left(q_{i}\right)_{j}=\left[\alpha_{k l}, \beta_{k l}\right]$ and $\sigma_{\delta}\left(e_{i}\right)_{j}=\omega_{k l}$ where $\alpha, \beta, \omega \in[0,1], k=1,2,3, \ldots, n$ and $l=1,2,3, \ldots, m$.

$$
\widetilde{M}_{n \times m}=\left[\left(\widetilde{a}_{i j}, a_{i j}\right)\right]_{n \times m}=\left[\begin{array}{llll}
\left(\widetilde{a}_{11}, a_{11}\right) & \left(\widetilde{a}_{12}, a_{12}\right) & \cdots & \left(\widetilde{a}_{1 m}, a_{1 m}\right) \\
\left(\widetilde{a}_{21}, a_{21}\right) & \left(\widetilde{a}_{22}, a_{22}\right) & \cdots & \left(\widetilde{a}_{2 m}, a_{2 m}\right) \\
\vdots & \vdots & \cdots & \vdots \\
\left(\widetilde{a}_{n 1}, a_{n 1}\right) & \left(\widetilde{a}_{n 2}, a_{n 2}\right) & \cdots & \left(\widetilde{a}_{n m}, a_{n m}\right)
\end{array}\right]
$$

or

$$
\widetilde{M}_{n \times m}=\left[\left(\widetilde{a}_{i j}, a_{i j}\right)\right]_{n \times m}=\left[\begin{array}{llll}
\left(\left[\alpha_{11}, \beta_{11}\right], \omega_{11}\right) & \left(\left[\alpha_{12}, \beta_{12}\right], \omega_{12}\right) & \cdots & \left(\left[\alpha_{1 m}, \beta_{1 m}\right], \omega_{1 m}\right) \\
\left(\left[\alpha_{21}, \beta_{21}\right], \omega_{21}\right) & \left(\left[\alpha_{22}, \beta_{22}\right], \omega_{22}\right) & \cdots & \left(\left[\alpha_{2 m}, \beta_{2 m}\right], \omega_{2 m}\right) \\
\vdots & \vdots & \cdots & \vdots \\
\left(\left[\alpha_{n 1}, \beta_{n 1}\right], \omega_{n 1}\right) & \left(\left[\alpha_{n 2}, \beta_{n 2}\right], \omega_{n 2}\right) & \cdots & \left(\left[\alpha_{n m}, \beta_{n m}\right], \omega_{n m}\right)
\end{array}\right] .
$$


Example 2.10. Suppose $U=\left\{u_{1}, u_{2}, u_{3}, u_{4}, u_{5}\right\}$ is a universal set where $u_{1}, u_{2}, u_{3}, u_{4}, u_{5}$ are representing five houses under consideration, and $E=\left\{e_{1}, e_{2}, e_{3}, e_{4}\right\}$ is the set of parameters, where $e_{i}$ stands for "beautiful", "large", "cheap" and "in water surroundings", respectively. Consider $A=\left\{e_{1}, e_{2}, e_{3}\right\} \subset E$ and $\delta$ is the mapping from $A$ to the set of all cubic subsets of power set of $U(\delta: A \rightarrow P(U))$. Consider a cubic soft set $(\delta, A)$ which describes the "attractiveness of houses" that is considering for purchase. Then cubic soft set $(\delta, A)$ is given as

$$
\begin{aligned}
(\delta, A) & =\left[\left(\widetilde{\mu}_{\delta}, \eta_{\delta}\right)\right]_{m \times n}, \text { where } \\
\widetilde{\mu}_{\delta}\left(e_{1}\right) & =\left\{\left(u_{1},[0.1,0.2]\right),\left(u_{2},[0.2,0.4]\right),\left(u_{3},[0.3,0.4]\right),\left(u_{4},[0.2,0.3]\right),\left(u_{5},[0.4,0.6]\right)\right\}, \\
\widetilde{\mu}_{\delta}\left(e_{2}\right) & =\left\{\left(u_{1},[0.2,0.3]\right),\left(u_{2},[0.1,0.5]\right),\left(u_{3},[0.3,0.4]\right),\left(u_{4},[0.2,0.6]\right),\left(u_{5},[0.5,0.7]\right)\right\}, \\
\widetilde{\mu}_{\delta}\left(e_{3}\right) & =\left\{\left(u_{1},[0.1,0.5]\right),\left(u_{2},[0.2,0.5]\right),\left(u_{3},[0.4,0.6]\right),\left(u_{4},[0.3,0.6]\right),\left(u_{5},[0.4,0.7]\right)\right\}, \\
\text { and } & \\
\eta_{\delta}\left(e_{1}\right) & =\left\{\left(u_{1}, 0.4\right),\left(u_{2}, 0.1\right),\left(u_{3}, 0.2\right),\left(u_{4}, 0.5\right),\left(u_{5}, 0.3\right)\right\}, \\
\eta_{\delta}\left(e_{2}\right) & =\left\{\left(u_{1}, 0.4\right),\left(u_{2}, 0.6\right),\left(u_{3}, 0.2\right),\left(u_{4}, 0.3,\right),\left(u_{5}, 0.1\right)\right\}, \\
\eta_{\delta}\left(e_{3}\right) & =\left\{\left(u_{1}, 0.2\right),\left(u_{2}, 0.7\right),\left(u_{3}, 0.8\right),\left(u_{4}, 0.1,\right),\left(u_{5}, 0.5\right)\right\} .
\end{aligned}
$$

We would represent this cubic soft set in matrix form as:

$$
\left[\begin{array}{llll}
([0.1,0.2], 0.66) & ([0.2,0.3], 0.66) & ([0.1,0.5], 0.66) & ([0.0,0.0], 0.0) \\
([0.2,0.4], 0.66) & ([0.1,0.5], 0.66) & ([0.2,0.5], 0.66) & ([0.0,0.0], 0.0) \\
([0.3,0.4], 0.66) & ([0.3,0.4], 0.66) & ([0.4,0.6], 0.66) & ([0.0,0.0], 0.0) \\
([0.2,0.3], 0.66) & ([0.2,0.6], 0.66) & ([0.3,0.6], 0.66) & ([0.0,0.0], 0.0) \\
([0.4,0.6], 0.66) & ([0.5,0.7], 0.66) & ([0.4,0.7], 0.66) & ([0.0,0.0], 0.0)
\end{array}\right] .
$$

Note. It is worth mentioning to add here that a cubic soft matrix is more general than a soft matrix (see example above).

Cubic Soft Null(Zero) Matrix: Let $\widetilde{U}=\left[\left(\widetilde{a_{i j}}, b_{i j}\right)\right] \in C S M_{m \times n}$. Then $\widetilde{U}$ is called a Zero Cubic Null Matrix denoted by $\widetilde{O}=[(\widetilde{0}, 0)]$ if $\widetilde{a_{i j}}=[0,0]$ and $b_{i j}=0$ for all $i$ and $j$.

Example 2.11.

$$
\widetilde{O}=\left(\begin{array}{cccc}
([0,0], 0) & ([0,0], 0) & ([0,0], 0) & ([0,0], 0) \\
([0,0], 0) & ([0,0], 0) & ([0,0], 0) & [0,0], 0) \\
[0,0], 0) & ([0,0], 0) & ([0,0], 0) & ([0,0], 0) \\
([0,0], 0) & ([0,0], 0) & ([0,0], 0) & ([0,0], 0)
\end{array}\right)
$$

Addition of intervals:

$$
\left[x_{1}, x_{2}\right]+\left[y_{1}, y_{2}\right]=\left[x_{1}+y_{1}, x_{2}+y_{2}\right], \text { where } x_{1}, x_{2}, y_{1}, y_{2} \in[0,1] .
$$

Subtraction of intervals:

$$
\left[x_{1}, x_{2}\right]-\left[y_{1}, y_{2}\right]=\left[x_{1}-y_{2}, x_{2}-y_{1}\right], \text { where } x_{1}, x_{2}, y_{1}, y_{2} \in[0,1] .
$$

Scalar multiplication of interval:

Let $R$ be any scalar number and $[x, y]$ be any interval then the scalar multiplication is given as

$$
R[x, y]=[R x, R y]
$$

\section{Applications of cubic soft matrix by using weighted arithmetic mean $\left(\widetilde{S}_{W A M}\right)$ in decision making}

In following we define arithmetic mean and weighted arithmetic mean of cubic soft matrix. 
Let $\widetilde{S}=\left[\left(\widetilde{a}_{i j}, a_{i j}\right)\right] \in C S M_{m \times n}$. Then Weighted Arithmetic Mean of Cubic Soft Matrix $\widetilde{S}$ of membership value $\widetilde{a}_{i j}$ and non-membership value $a_{i j}$ denoted by $\widetilde{S}_{W A M}$ is defined as

$$
\widetilde{S}_{W A M}=\left[\left(\frac{\sum_{j=1}^{n} \widetilde{a}_{i j}}{n}, \frac{\sum_{j=1}^{n} a_{i j}}{n}\right)\right]
$$

when weights are same.

Let $\widetilde{S}=\left[\left(\widetilde{a}_{i j}, a_{i j}\right)\right] \in C S M_{m \times n}$. Then Weighted Arithmetic Mean of Cubic Soft Matrix $\widetilde{S}$ for membership and non-membership value denoted by $\widetilde{S}_{W A M}$ is defined as

$$
\widetilde{S}_{W A M}=\left[\left(\frac{\sum_{j=1}^{n} w_{j} \widetilde{a}_{i j}}{\sum_{j=1}^{n} w_{j}}, \frac{\sum_{j=1}^{n} w_{j} a_{i j}}{\sum_{j=1}^{n} w_{j}}\right)\right],
$$

where $w_{j}$ are respective weights for $j=1,2,3, \ldots, n$.

Algorithm 3.1. Step-1: Select the set of parameters.

Step-2: Construct the cubic soft matrix for the set of parameters.

Step-3: Calculate the weighted arithmetic mean of membership and non-membership value of cubic soft matrix as $\widetilde{S}_{W A M}$.

Step-4: Choose the object with the highest membership value.

In case of tie i.e, when at the same time more than one object having same highest membership value, choose the object with the highest membership value as well as the lowest non-membership value.

Example 3.2. Let we have $U=\left\{h_{1}, h_{2}, h_{3}, h_{4}, h_{5}\right\}$ as a set of universe where $h_{1}, h_{2}, h_{3}, h_{4}, h_{5}$ represents the five different types of mobile phones and let the set of parameters be

$$
E=\left\{e_{1} \text { (battery timing), } e_{2} \text { (camera quality), } e_{3} \text { (speed) }\right\} .
$$

Suppose Mr. X wants to buy a mobile phone. Then cubic soft set based on the above parameters is as follow

$$
(F, E)=\left\{\left(e_{1}, f\left(e_{1}\right)\right),\left(e_{2}, f\left(e_{2}\right)\right),\left(e_{3}, f\left(e_{3}\right)\right)\right\},
$$

where

$$
\begin{aligned}
& \left.f\left(e_{1}\right)=\left(h_{1},([0.4,0.5], 0.1)\right),\left(h_{2},([0.1,0.9], 0.2)\right),\left(h_{3},([0.3,0.5], 0.4)\right),\left(h_{4},([0.4,0.6], 0.3)\right),\left(h_{5},([0.1,0.6], 0.9)\right)\right\}, \\
& f\left(e_{2}\right)=\left\{\left(h_{1},([0.2,0.3], 0.4)\right),\left(h_{2},([0.1,0.5], 0.3)\right),\left(h_{3},([0.3,0.6], 0.5)\right),\left(h_{4},([0.2,0.5], 0.4)\right),\left(h_{5},([0.1,0.6], 0.9)\right)\right\}, \\
& f\left(e_{3}\right)=\left\{\left(h_{1},([0.3,0.4], 0.2)\right),\left(h_{2},([0.1,0.3], 0.4)\right),\left(h_{3},([0.1,0.7], 0.6)\right),\left(h_{4},([0.3,0.5], 0.1)\right),\left(h_{5},([0.4,0.8], 0.2)\right)\right\},
\end{aligned}
$$

and the corresponding cubic soft matrix is

$$
\widetilde{S}=\left[\begin{array}{lll}
([0.4,0.5], 0.1) & ([0.2,0.3], 0.4) & ([0.3,0.4], 0.2) \\
([0.1,0.9], 0.2) & ([0.1,0.5], 0.3) & ([0.1,0.3], 0.4) \\
([0.3,0.5], 0.4) & ([0.3,0.6], 0.5) & ([0.1,0.7], 0.6) \\
([0.4,0.6], 0.3) & ([0.2,0.5], 0.4) & ([0.3,0.5], 0.1) \\
([0.1,0.6], 0.9) & ([0.3,0.6], 0.8) & ([0.4,0.8], 0.2)
\end{array}\right]
$$

The arithmetic mean of above cubic soft matrix is 


$$
\widetilde{S}_{A M}=\left[\begin{array}{l}
([0.3,0.4], 0.23) \\
([0.1,0.56], 0.3) \\
([0.23,0.6], 0.5) \\
([0.3,0.53], 0.26) \\
([0.26,0.66], 0.63)
\end{array}\right]
$$

If we prefer battery timing of the mobile phone and $0.7,0.2,0.1$ are weights used for the parameters "battery timing" , "camera quality" , "best speed", respectively, then weighted arithmetic mean is

$$
\widetilde{S}_{W A M}=\left[\begin{array}{l}
([0.35,0.45], 0.17) \\
([0.1,0.76], 0.24) \\
([0.28,0.54], 0.44) \\
([0.35,0.57], 0.3) \\
([0.17,0.62], 0.81)
\end{array}\right] .
$$

From the above result we get $[0.1,0.56]$ as the highest membership value if we use equal preference, which shows that the most appropriate mobile phone for Mr. $X$ is $h_{2}$. But also we conclude that if we use more preference on "battery timing" than other parameters (camera quality, speed), then the most appropriate mobile phone for Mr. $X$ is $h_{2}$.

\section{Applications of cubic soft matrix in medical diagnosis}

Definition 4.1. The membership value matrix corresponding to the matrix $\widetilde{A}$ is $M V(\widetilde{A})=[\delta i j]_{m \times n}$, where $\delta i j=\mu_{i j}-\gamma_{i j} \forall i=1,2, \ldots, m$ and $j=1,2, \ldots, n . \mu_{i j}$ and $\gamma_{i j}$ represent the fuzzy membership function and fuzzy reference function respectively of $u_{i}$ in the fuzzy set $F\left(e_{j}\right)$.

Definition 4.2. Let $\widetilde{A}=\left[\left(\widetilde{a_{i j}}, a_{i j}\right)\right]_{m \times n}$, where $\widetilde{a_{i j}}=\left[a_{1}, a_{2}\right]$, such that $0 \leq a_{1} \leq a_{2} \leq 1$ and $a_{i j} \in[0,1]$. Also, let $\widetilde{B}=\left[\left(\widetilde{b_{j k}}, b_{j k}\right)\right]_{n \times p}$, where $\widetilde{b_{j k}}=\left[b_{1}, b_{2}\right]$ such that $0 \leq b_{1} \leq b_{2} \leq 1$. Then

$$
d_{j k}=\operatorname{length}\left(\left[b_{1}, b_{2}\right]\right)-b_{j k},
$$

gives the membership value. We now define $\widetilde{A} \cdot \widetilde{B}$, the product of $\widetilde{A}$ and $\widetilde{B}$ as $\widetilde{A} \cdot \widetilde{B}=\left[c_{i k}\right]_{m \times p}=\left[\max -\min \left(\widetilde{a_{i j}}, \widetilde{b_{j k}}\right), \min -\max \left(a_{i j}, b_{j k}\right)\right]_{m \times p}, 1 \leq i \leq m, 1 \leq k \leq p$ for all $j=1,2, \cdots, n$.

Definition 4.3. Cubic soft universal matrix: A cubic soft matrix $\widetilde{A}=\left[\left(\widetilde{a}_{i j}, a_{i j}\right)\right]_{m \times n}$ is called cubic soft universal matrix and denoted by $\widetilde{U}$ if $\widetilde{a}_{i j}=[1,1]$ and $a_{i j}=1$, for all $i$ and $j$.

Example 4.4.

$$
\widetilde{U}=\left(\begin{array}{llll}
([1,1], 1) & ([1,1], 1) & ([1,1], 1) & ([1,1], 1) \\
([1,1], 1) & ([1,1], 1) & ([1,1], 1) & ([1,1], 1) \\
([1,1], 1) & ([1,1], 1) & ([1,1], 1) & ([1,1], 1) \\
([1,1], 1) & ([1,1], 1) & ([1,1], 1) & ([1,1], 1)
\end{array}\right)_{4 \times 4} .
$$

Definition 4.5. Cubic soft complement matrix: If $\widetilde{A}=\left[\left(\widetilde{a}_{i j}, a_{i j}\right)\right]_{m \times n}$ is a cubic soft matrix, then complement of $\widetilde{A}$ is $\widetilde{A}^{C}=\left[\left(\widetilde{a}_{i j}^{C}, a_{i j}^{C}\right)\right]_{n \times m}$ and defined as

$$
\widetilde{a}_{i j}^{C}=\left[1-\beta_{R C}, 1-\alpha_{R C}\right] \text { and } a_{i j}^{C}=1-\omega_{R C}, \text { for all } i \text { and } j,
$$

where $\widetilde{a}_{i j}=\widetilde{\rho}_{\delta}\left(e_{i}\right)_{j}=\left[\alpha_{R C}, \beta_{R C}\right]$ and $a_{i j}=\widetilde{\rho}_{\delta}\left(e_{i}\right)_{j}=\omega_{R C}$ such that $\alpha, \beta, \omega \in[0,1]$ and $R=1,2,3, \ldots m$ and $C=1,2,3, \ldots n$.

Consider $\widetilde{A}$ is a cubic soft matrix such that

$$
\widetilde{A}=\left(\begin{array}{ll}
([0.66,0.88], 0.66) & ([0.77,0.88], 0.77) \\
([0.55,0.66], 0.55) & ([0.88,0.99], 0.88)
\end{array}\right)_{2 \times 2} .
$$


Then complement $\widetilde{A}^{C}$ of matrix $\widetilde{A}$ is given as:

$$
\widetilde{A}^{C}=\left(\begin{array}{ll}
([0.12,0.34], 0.34) & ([0.12,0.23], 0.23) \\
([0.34,0.45], 0.45) & ([0.01,0.12], 0.12)
\end{array}\right)_{2 \times 2} .
$$

\section{Methodology}

Here we will present an algorithm for diagnosis the disease by using the cubic soft matrices. Suppose $P=\left\{p_{1}, p_{2}, p_{3}, \ldots . ., p_{m}\right\}$ is a set of $m$ patients having a set of $n$ symptoms $S=\left\{s_{1}, s_{2}, s_{3}, \ldots ., s_{n}\right\}$ related to a set of $k$ diseases $D=\left\{d_{1}, d_{2}, d_{3}, \ldots . ., d_{k}\right\}$.

To diagnose which patient has what disease we will apply cubic soft set theory to develop a technique through Sanchez's method.

For this, construct a cubic soft set $(\widetilde{F}, D)$ over $S$. This cubic soft set gives a relation matrix $\widetilde{A}$, called symptom-disease matrix, where each element denote the weight of the symptoms for a certain disease. Also, its complement $(F, D)^{c}$ gives another relation matrix $\widetilde{A}^{c}$ called non-symptom disease matrix. We call the matrices $\widetilde{A}$ and $\widetilde{A}^{c}$ as medical knowledge of cubic soft set.

Then construct another cubic soft set $(G, S)$ over $P$. This cubic soft set gives a relation matrix $\widetilde{B}$, called patient symptom matrix. Similarly, its complement $(G, S)^{c}$ gives the relation matrix $\widetilde{B}^{c}$ called patient non-symptom matrix.

Thus the general form of $\widetilde{A}$ and $\widetilde{A}^{C}$ are

$$
\begin{gathered}
\widetilde{A}=\left[\begin{array}{llll}
\left(\widetilde{a_{11}}, a_{11}\right) & \left(\widetilde{a_{12}}, a_{12}\right) & \cdots & \left(\widetilde{a_{1 n}}, a_{1 n}\right) \\
\left(\widetilde{a_{21}}, a_{21}\right) & \left(\widetilde{a_{22}}, a_{22}\right) & \cdots & \left(\widetilde{a_{2 n}}, a_{2 n}\right) \\
\vdots & \vdots & \cdots & \vdots \\
\left(\widetilde{a_{m 1}}, a_{m 1}\right) & \left(\widetilde{a_{m 2}}, a_{m 2}\right) & \cdots & \left(\widetilde{a_{m n}}, a_{m n}\right)
\end{array}\right], \\
\widetilde{A}^{C}=\left[\begin{array}{llll}
\left(I-\widetilde{a_{11}}, 1-a_{11}\right) & \left(I-\widetilde{a_{12}}, 1-a_{12}\right) & \cdots & \left(I-\widetilde{a_{1 n}}, 1-a_{1 n}\right) \\
\left(I-\widetilde{a_{21}}, 1-a_{21}\right) & \left(I-\widetilde{a_{22}}, 1-a_{22}\right) & \cdots & \left(I-\widetilde{a_{2 n}}, 1-a_{2 n}\right) \\
\vdots & \vdots & \cdots & \vdots \\
\left(I-\widetilde{a_{m 1}}, 1-a_{m 1}\right) & \left(I-\widetilde{a_{m 2}}, 1-a_{m 2}\right) & \cdots & \left(I-\widetilde{a_{m n}}, 1-a_{m n}\right)
\end{array}\right],
\end{gathered}
$$

and the general form of $\widetilde{B}$ and $\widetilde{B}^{c}$

$$
\begin{gathered}
\widetilde{B}=\left[\begin{array}{llll}
\left(\widetilde{b_{11}}, b_{11}\right) & \left(\widetilde{b_{12}}, b_{12}\right) & \cdots & \left(\widetilde{b_{1 n}}, b_{1 n}\right) \\
\left(\widetilde{b_{21}}, b_{21}\right) & \left(\widetilde{b_{22}}, b_{22}\right) & \cdots & \left(\widetilde{b_{2 n}}, b_{2 n}\right) \\
\vdots & \vdots & \cdots & \vdots \\
\left(\widetilde{b_{m 1}}, b_{m 1}\right) & \left(\widetilde{b_{m 2}}, b_{m 2}\right) & \cdots & \left(\widetilde{b_{m n}}, b_{m n}\right)
\end{array}\right], \\
\widetilde{B}^{C}=\left[\begin{array}{llll}
\left(I-\widetilde{b_{11}}, 1-b_{11}\right) & \left(I-\widetilde{b_{12}}, 1-b_{12}\right) & \cdots & \left(I-\widetilde{b_{1 n}}, 1-b_{1 n}\right) \\
\left(I-\widetilde{b_{21}}, 1-b_{21}\right) & \left(I-\widetilde{b_{22}}, 1-b_{22}\right) & \cdots & \left(I-\widetilde{b_{2 n}}, 1-b_{2 n}\right) \\
\vdots & \vdots & \cdots & \vdots \\
\left(I-\widetilde{b_{m 1}}, 1-b_{m 1}\right) & \left(I-\widetilde{b_{m 2}}, 1-b_{m 2}\right) & \cdots & \left(I-\widetilde{b_{m n}}, 1-b_{m n}\right)
\end{array}\right],
\end{gathered}
$$

where $I$ is unit interval.

By using the definition, we get the matrices $\widetilde{T}_{1}=\widetilde{B} \cdot \widetilde{A}$ and $\widetilde{T}_{2}=\widetilde{B} \cdot \widetilde{A}^{c}$ called the patient symptom disease matrix and patient symptom non-disease matrix, respectively, similarly we get $\widetilde{T}_{3}=\widetilde{B}^{c} . \widetilde{A}$ and $\widetilde{T}_{4}=\widetilde{B}^{c} \cdot \widetilde{A}^{c}$ called the patient non-symptom disease matrix and patient non-symptom non-disease matrix, respectively.

Using Definition 4.31, we obtain the corresponding membership value matrices $M V\left(\widetilde{T}_{1}\right), M V\left(\widetilde{T}_{2}\right), M V\left(\widetilde{T}_{3}\right)$ and $M V\left(\widetilde{T}_{4}\right)$. We calculate the diagnosis score $\widetilde{S}_{T_{1}}$ and $\widetilde{S}_{T_{2}}$ for and against the disease respectively as 


$$
\widetilde{S}_{T_{1}}=\left[\gamma\left(\widetilde{T}_{1}\right)_{i j}\right]_{m \times n}, \text { where } \gamma\left(\widetilde{T}_{1}\right)_{i j}=\delta\left(\widetilde{T}_{1}\right)_{i j}-\delta\left(\widetilde{T}_{3}\right)_{i j}
$$

and

$$
\widetilde{S}_{T_{2}}=\left[\gamma\left(\widetilde{T}_{2}\right)_{i j}\right]_{m \times n}, \text { where } \gamma\left(\widetilde{T}_{2}\right)_{i j}=\delta\left(\widetilde{T}_{2}\right)_{i j}-\delta\left(\widetilde{T}_{4}\right)_{i j} .
$$

Now, if $\max _{j}\left[\widetilde{S}_{T_{1}}\left(p_{i}, d_{j}\right)-\widetilde{S}_{T_{2}}\left(p_{i}, d_{j}\right)\right]$, then we have

Algorithm 5.1. Step-1: Input the cubic soft set $(F, D)$ and compute $(F, D)^{c}$. Compute the corresponding matrices $\widetilde{A}$ and $\widetilde{A}^{c}$. $\widetilde{B}^{c}$.

Step-2: Input the cubic soft set $(G, S)$ and compute $(G, S)^{c}$. Compute the corresponding matrices $\widetilde{B}$ and

Step-3: Compute $T_{1}=\widetilde{B} \cdot \widetilde{A}, T_{2}=\widetilde{B} \cdot \widetilde{A}^{c}, T_{3}=\widetilde{B}^{c} \cdot \widetilde{A}$, and $T_{4}=\widetilde{B}^{c} \cdot \widetilde{A}^{c}$.

Step-4: Compute the corresponding membership value matrices $M V\left(\widetilde{T}_{1}\right), M V\left(\widetilde{T}_{2}\right), M V\left(\widetilde{T}_{3}\right)$ and $M V\left(\widetilde{T}_{4}\right)$.

Step-5: Compute the diagnosis score $S_{\widetilde{T}_{1}}$ and $S_{\widetilde{T}_{2}}$.

Step-6: Find $S_{k}=\max _{j}\left[S_{\widetilde{T}_{2}}\left(p_{i}, d_{j}\right)-S_{\widetilde{T}_{1}}\left(p_{i}, d_{j}\right)\right]$.

Then we conclude that the patient $p_{i}$ is suffering from the disease $d_{k}$. If $S_{k}$ has more than one similar value, then go to step-1 and repeat the process by reassessing the symptoms for the patient.

Example 5.2. Suppose that there are three patients John, George and Albert in a hospital those who intake over dosage for sensual pleasure which will affect the brain cells lead to the symptoms of hysteria. Then the patient who used sleeping pills will have the side affect of headache and stomach pain, then the patient who take birth control pills will have side effect of depression and stroke. We consider the set $S=\left\{s_{1}, s_{2}, s_{3}\right\}$ as universal set where $s_{1}, s_{2}, s_{3}$ represent symptoms of hysteria, headache and stomach pain, depression and stroke problems, respectively, and the set $D=\left\{d_{1}, d_{2}\right\}$ where $d_{1}$ and $d_{2}$ represent the parameters of side effect in the human body, particularly brain and heart problem disease, respectively.

Suppose,

$$
\begin{aligned}
(F, D) & =\left\{F\left(d_{1}\right)=\left\{\left(s_{1},([0.3,0.4], 0.2)\right),\left(s_{2},([0.2,0.6], 0.7)\right),\left(s_{3},([0.3,0.6], 0.5)\right)\right\},\right. \\
F\left(d_{2}\right) & \left.=\left\{\left(s_{1},([0.4,0.8], 0.3)\right),\left(s_{2},([0.5,0.6], 0.4)\right),\left(s_{3},([0.7,0.8], 0.5)\right)\right\}\right\},
\end{aligned}
$$

and complement of $(F, D)$ is defined as

$$
\begin{aligned}
(F, D)^{c} & =\left\{F^{c}(d 1)=\left\{\left(s_{1},([0.6,0.7], 0.8)\right),\left(s_{2},([0.4,0.8], 0.3)\right),\left(s_{3},([0.4,0.7], 0.5)\right)\right\},\right. \\
F^{c}\left(d_{2}\right) & \left.=\left\{\left(s_{1},([0.2,0.6], 0.7)\right),\left(s_{2},([0.4,0.5], 0.6)\right),\left(s_{3},([0.2,0.3], 0.5)\right)\right\}\right\} .
\end{aligned}
$$

Now, the cubic fuzzy soft matrices $A$ and $A^{c}$ of cubic fuzzy soft set $(F, D)$ and its compliment set $(F, D)^{c}$, respectively, are given in the following.

$$
\begin{gathered}
\widetilde{A}=\left[\begin{array}{ll}
([0.3,0.4], 0.2) & ([0.4,0.8], 0.3) \\
([0.2,0.6], 0.7) & {[(0.5,0.6], 0.4)} \\
([0.3,0.6], 0.5) & ([0.7,0.8], 0.5)
\end{array}\right], \\
\widetilde{A}^{c}=\left[\begin{array}{cc}
([0.6,0.7], 0.8) & ([0.2,0.6], 0.7) \\
([0.4,0.8], 0.3 & ([0.4,0.5], 0.6) \\
([0.4,0.7], 0.5) & ([0.2,0.3], 0.5)
\end{array}\right] .
\end{gathered}
$$

Next suppose,

$$
\begin{aligned}
(G, S) & =\left\{G\left(s_{1}\right)=\left\{\left(p_{1},([0.4,0.7], 0.2)\right),\left(p_{2},([0.3,0.6], 0.4)\right),\left(p_{3},([0.5,0.8], 0.3)\right)\right\},\right. \\
G\left(s_{2}\right) & =\left\{\left(p_{1},([0.7,0.8], 0.5)\right),\left(p_{2},([0.1,0.4], 0.6)\right),\left(p_{3},([0.2,0.4], 0.7)\right)\right\}, \\
G\left(s_{3}\right) & \left.=\left\{\left(p_{1},([0.1,0.3], 0.8)\right),\left(p_{2},([0.7,0.9], 0.1)\right),\left(p_{3},([0.2,0.6], 0.5)\right)\right\}\right\},
\end{aligned}
$$


and complement of $(G, S)$ is given by

$$
\begin{aligned}
(G, S)^{c} & =\left\{G^{c}\left(s_{1}\right)=\left\{\left(p_{1},([0.3,0.6], 0.8)\right),\left(p_{2},([0.4,0.7], 0.6)\right),\left(p_{3},([0.2,0.5], 0.7)\right)\right\}\right. \\
G^{c}\left(s_{2}\right) & =\left\{\left(p_{1},([0.2,0.3], 0.5)\right),\left(p_{2},([0.6,0.9], 0.4)\right),\left(p_{3},([0.6,0.8], 0.3)\right)\right\} \\
G^{c}\left(s_{3}\right) & \left.=\left\{\left(p_{1},([0.7,0.9], 0.2)\right),\left(p_{2},([0.1,, 0.3], 0.9)\right),\left(p_{3},([0.4,0.8], 0.5)\right)\right\}\right\} .
\end{aligned}
$$

Now, the cubic soft matrices $\widetilde{B}$ and $\widetilde{B}^{c}$ of cubic soft sets $(G, S)$ and its compliment set $(G, S)^{c}$, respectively, are given in the following.

$$
\begin{gathered}
\widetilde{B}=\left[\begin{array}{lll}
([0.4,0.7], 0.2) & ([0.7,0.8], 0.5) & ([0.1,0.3], 0.8) \\
([0.3,0.6], 0.4) & ([0.1,0.4], 0.6) & ([0.7,0.9], 0.1) \\
([0.5,0.8], 0.3) & ([0.2,0.4], 0.7) & ([0.2,0.6], 0.5)
\end{array}\right], \\
\widetilde{B}^{c}=\left[\begin{array}{lll}
([0.4,0.6], 0.8) & ([0.2,0.3], 0.5) & ([0.7,0.9], 0.8) \\
([0.4,0.7], 0.6) & ([0.6,0.9], 0.4) & ([0.1,0.3], 0.9) \\
([0.2,0.5], 0.7) & ([0.6,0.8], 0.3) & ([0.4,0.8], 0.5)
\end{array}\right] .
\end{gathered}
$$

Now,

$$
\begin{gathered}
\widetilde{T_{1}}=\widetilde{B} \cdot \widetilde{A}=\left[\begin{array}{ll}
([0.1,0.3], 0.2) & ([0.4,0.7], 0.3) \\
([0.1,0.4], 0.4) & ([0.3,0.6], 0.4) \\
([0.3,0.6], 0.3) & ([0.5,0.8], 0.3)
\end{array}\right], \\
\widetilde{T_{2}}=\widetilde{B} \cdot \widetilde{A}^{c}=\left[\begin{array}{ll}
([0.1,0.3], 0.5) & ([0.4,0.7], 0.6) \\
([0.1,0.4], 0.4) & ([0.3,0.6], 0.7) \\
([0.4,0.7], 0.5) & ([0.5,0.8], 0.8)
\end{array}\right], \\
\widetilde{T_{3}}=\widetilde{B}^{c} . \widetilde{A}=\left[\begin{array}{ll}
([0.7,0.9], 0.5) & ([0.3,0.6], 0.5) \\
([0.6,0.9], 0.7) & ([0.4,0.7], 0.5) \\
([0.3,0.6], 0.6) & ([0.2,0.5], 0.4)
\end{array}\right], \\
\widetilde{T_{4}}=\widetilde{B}^{c} \cdot \widetilde{A}^{c}=\left[\begin{array}{ll}
([0.7,0.9], 0.5) & ([0.3,0.6], 0.5) \\
([0.6,0.9], 0.4) & ([0.4,0.7], 0.6) \\
([0.4,0.7], 0.3) & ([0.2,0.5], 0.5)
\end{array}\right] .
\end{gathered}
$$

Now, the membership value matrices of above cubic matrices are following

$$
\begin{array}{ll}
M V\left(\widetilde{T_{1}}\right)=\left[\begin{array}{ll}
0 & 0 \\
0.1 & 0.1 \\
0 & 0
\end{array}\right], & M V\left(\widetilde{T_{2}}\right)=\left[\begin{array}{ll}
0.3 & 0.3 \\
0.1 & 0.4 \\
0.2 & 0.5
\end{array}\right], \\
M V\left(\widetilde{T_{3}}\right)=\left[\begin{array}{ll}
0.3 & 0.2 \\
0.4 & 0.2 \\
0.3 & 0.1
\end{array}\right], & M V\left(\widetilde{T_{4}}\right)=\left[\begin{array}{ll}
0.3 & 0.2 \\
0.1 & 0.3 \\
0 & 0.2
\end{array}\right] .
\end{array}
$$

Now, the diagnosis score $S_{\widetilde{T_{1}}}$ and $S_{\widetilde{T_{2}}}$ for and against the disease are following

$$
S_{\widetilde{T_{1}}}=\left[\begin{array}{ll}
-0.3 & -0.2 \\
-0.3 & -0.1 \\
-0.3 & -0.1
\end{array}\right], \quad S_{\widetilde{T_{2}}}=\left[\begin{array}{ll}
0 & 0.1 \\
0 & 0.1 \\
0.2 & 0.3
\end{array}\right] .
$$

Now, the difference for and against the disease we have

$$
S_{\widetilde{T_{2}}}-S_{\widetilde{T_{1}}}=\left[\begin{array}{cc}
0.3 & 0.3 \\
0.3 & 0.2 \\
0.5 & 0.4
\end{array}\right]
$$




\section{Conclusion}

In this paper, we introduced cubic soft matrices (CSM) and defined some arithmetic weighted arithmetic means on these matrices. We discussed several properties of CSM and investigated some results. In addition, we gave applications of these matrices in decision making problems and medical diagnosis.

\section{Acknowledgment}

This work is financially supported by the Higher Education Commission of Pakistan (Grant No: 7750/Federal/ NRPU/R\&D/HEC/ 2017).

Conflict of Interest. The authors declare that there is no conflict of interest regarding the publication of this article.

\section{References}

[1] K. Atanassov, Intuitionistic fuzzy sets, Fuzzy Sets and Systems, 20 (1986), 87-96.

[2] K. Atanassov, Operators over interval valued intuitionistic fuzzy sets, Fuzzy Sets and Systems, 64 (1994), 159-174.

[3] K.V. Babitha, S.J. John, Generalized intuitionistic fuzzy soft sets and its applications, General Mathematics Notes, 7 (2011), 1-14.

[4] M.J. Borah, T.J. Neog, D.K. Sut, Fuzzy soft matrix theory and its decision making, International Journal of Modern Engineering Research, 2(2) (2012), 121-127.

[5] N. Cagman, S. Enginoglu, Soft matrix theory and its decision making, Journal Computers and Mathematics with Applications, 59(10) (2010), 3308-3314.

[6] N. Cagman, S. Enginoglu, Fuzzy soft matrix theory and its application in decision making, Iranian Journal of Fuzzy Systems, 9(1) (2012), 109-119.

[7] D. Chen, E.C.C. Tsang, D.S. Yeung, X. Wang, The parameterization reduction of soft sets and its applications, Computers and Mathematics with Applications, 49 (2005), 757-763.

[8] S.K. De, R. Biswas, A.R. Roy, An application of intuitionistic fuzzy sets in medical diagnostic, Fuzzy Sets and Systems, 117(2) (2001), 209-213.

[9] I. Deli, N. Cagman, Intuitionistic fuzzy parameterized soft set theory and its decision making, Applied Soft Computing, 28 (2015), 109-113.

[10] W.L. Gau, D.J. Buehrer, Vague sets, IEEE Transactions on Systems, Man, and Cybernetics, 23(2) (1993), 610-614.

[11] M.B. Gorzalzany, A method of inference in approximate reasoning based on interval-valued fuzzy sets, Fuzzy Sets and Systems, 21 (1987), 1-17.

[12] Y.B. Jun, M. Mohseni Takallo, Commutative MBJ-neutrosophic ideals of BCK-algebras, Journal of Algebraic Hyperstructures and Logical Algebras, 2(1) (2021), 69-81.

[13] Y.B. Jun, S.Z. Song, Crossing cubic ideals of BCK/BCI-algebras, Journal of Algebraic Hyperstructures and Logical Algebras, 2(1) (2021), 17-31.

[14] P. K. Maji, R. Biswas, A.R. Roy, Fuzzy soft sets, Journal of Fuzzy Mathematics, 9(3) (2001), 589-602.

[15] P.K. Maji, A.R. Roy, R. Biswas, An application of soft sets in a decision making problem, Computers and Mathematics with Applications, 44(8-9) (2002), 1077-1083. 
[16] D.A. Molodtsov, Soft set theory-first results, Computers and Mathematics with Applications, 37 (1999), $19-31$.

[17] G. Muhiuddin, A.M. Al-roqi, Cubic soft sets with applications in BCK/BCL-algebras, Annals of Fuzzy Mathematics and Informatics, In press.

[18] Z. Pawlak, Rough sets, International Journal of Information and Computer Sciences, 11 (1982), 341356.

[19] P. Rajarajeswari, P. Dhanalakshmi, Intuitionistic fuzzy soft matrix theory and its application in decision making, International Journal of Engineering Research and Technology, 2 (2013), 1100-1111.

[20] E. Sanchez, Inverse of fuzzy relations, application to possibility distributions and medical diagnosis, Fuzzy Sets and Systems, 2(1) (1979), 75-86.

[21] Z. Xiao, Y. Li, B. Zhong, X. Yang, Research on synthetically evaluating method for business competitive capacity based on soft set, Statistical Research, (2003), 52-54.

[22] Y. Yang, C. Ji, Fuzzy soft matrices and their applications, Artificial Intelligence and Computational Intelligence Lecture Notes in Computer Science, 7002 (2011), 618-627.

[23] X. Yang, D. Yu, J. Yang, C. Wu, Generalization of soft set theory: From crisp to fuzzy case, Fuzzy Information and Engineering, 40 (2007), 345-354.

[24] L.A. Zadeh, Fuzzy sets, Information and Control, 8 (1965), 338-353. 